\title{
LA ORIENTACIÓN DE LAS PRÁCTICAS DE LABORATORIO COMO INVESTIGACIÓN: UN EJEMPLO ILUSTRATIVO
}

\author{
GIL PÉREZ, D. Y VALDÉS CASTRO, P. ${ }^{2}$ \\ 1 Departamento de Didáctica de las Ciencias Experimentales. Universitat de València. \\ ${ }^{2}$ Departamento de Física. Instituto Superior Pedagógico de La Habana.
}

\section{SUMMARY}

This paper offers a detailed example of transformation of a science practical work, from the usual simple recipe into an open problematic situation capable of favouring pupils' research.

\section{INTRODUCCIÓN}

Los estudios sobre prácticas de laboratorio están adquiriendo estos últimos años un nuevo impulso (Caamaño, Carrascosa y Oñorbe, 1994; Hodson, 1994) y generando un amplio consenso en torno a su orientación como actividad investigativa (Gil et al., 1991; González, 1992; Hodson, 1992 y 1993; Tamir y Garçía, 1992; Grau, 1994; Lillo, 1994; Watson, 1994).

Está contribuyendo a ello la comprensión de que la atención casi exclusiva hacia las preconcepciones que se produjo durante la década de los ochenta supone un grave reduccionismo que podría explicar las limitaciones de las estrategias de cambio conceptual (Duschi y Gitomer, 1991).

Se ha empezado así a tener en cuenta que la construcción de conocimientos científicos tiene exigencias metodológicas y epistemológicas a las que es preciso prestar atención explícita (Gil y Carrascosa, 1985; Hashweh 1986; Duschl y Gitomer, 1991; Wheatley, 1991; Hodson, 1992b). Y ello ha proporcionado nuevo sentido e interés a las investigaciones sobre resolución de problemas đe lápiz y papel o sobre las prácticas de laboratorio que aquí nos ocupan, provocando su reorientación para que dejen de ser meras ilustraciones de los conocimientos transmitidos y pasen a constituir actividades de investigación.

El consenso existente en torno a la necesidad de esta reorientación merece ser resaltado, pero es preciso ir más allá y mostrar de forma concreta, con ejemplos ilustrativos, lo que cada cual entiende por eprácticas como investigaciones». En caso contrario, corremos el peligro de que dicha expresión no pase de ser un simple eslogan, atractivo pero escasamente operativo, mientras la generalidad del profesorado continúa prestando escasa atención a las prácticas de laboratorio (Nieda, 1994).

Si queremos avanzar realmente en la transformación de las prácticas de laboratorio, es necesario analizar cuidadosamente las propuestas concretas, llevarlas al aula y contrastar su validez, como han comenzado a hacer diversos autores (Payá, 1991; Gil, Navarro y González, 1993; González, 1994; Salinas, 1994). Ello permite constatar indudables convergencias, pero también-lo que noes menos importante- diferencias que deben ser discutidas para hacer posible Ia profundización del consenso o un mejor conocimiento de las distintas aiternativas. 
El presente trabajo - fruto de la colaboración de dos equipos con diferentes tradiciones en el tratamiento de las prácticas de iaboratorio- pretende contribuir a dicho objetivo presentando la remodelación de una práctica habitual para convertirla en lo que pensamos que se aproxima a una investigación dirigida. Antes, sin embargo, expondremos muy brevemente las características generales que, según nuestra opinión consensuada, deberían tener las prácticas de laboratorio para que podamos considerarlas como una actividad investigativa.

\section{¿DE QUÉ CLASE DE INVESTIGACIÓN ESTAMOS HABLANDO?}

La revista Investigación en la Escuela, en su número 23 (1994), ha abierto un debate en torno a las relaciones entre conocimiento escolar y sus relaciones con el conocimiento cotidiano y el conocimiento científico, tratando de salir al paso de identificaciones abusivas, que se traducen en visiones simplistas y deformadas de la actividad científica. Como señalábamos en una contribución a dicho debate (Gil, 1994), nuestra propuesta de investigación dirigida no toma como referencia la actividad de los científicos que trabajan en la frontera del conocimiento, sino que utiliza la metáfora de los investigadores noveles (Gil, 1993), cuya actividad consiste en la réplica de investigaciones bien conocidas por el experto que dirige y apoya su trabajo. Estas réplicas de investigaciones han de ajustarse, sin embargo, a las estrategias del trabajo científico, sin caer en los reduccionismos y deformaciones que una abundante investigación ha detectado en la "epistemología espontánea" de los profesores (Désautels et al., 1993; Gi1, 1993; Hodson, 1993; Meichstry, 1993; Praia y Cachapuz, 1994), hasta el punto que, como señalan Bell y Pearson (1992), «empieza a comprenderse que, si se quiere cambiar lo que los profesores y estudiantes hacemos en las clases de ciencias, es preciso previamente modificar la eepistemología de los profesores» y salir al paso de visiones deformadas sobre el trabajo científico que actúan como auténticos obstáculos. No podemos aquí extendernos en el análisis de estas deformaciones ni en la fundamentación de una visión epistemológicamente correcta (Gil et al., 1991) y nos limitaremos, pues, a enunciar el rechazo, ampliamente compartido por los investigadores, a cualquier intento de reducir la complejidad y riqueza de la actividad científica a una receta simplista.

Desde este punto de vista, una práctica de laboratorio que pretenda aproximarse a una investigación ha de dejar de ser un trabajo exclusivamente experimental e integrar muchos otros aspectos de la actividad cientifica igualmente esenciales. De forma muy resumida nos referiremos, a continuacion, a una serie de aspectos cuya presencia consideramos fundamental para poder hablar de una orientación investigativa de las prácticas. Hemos agrupado dichos aspectos en 10 puntos, pero nos apresuramos a señalar que no constituyen ningún algoritmo a seguir linealmente, sino un recordatorio de la extraordi- naria riqueza de la actividad científica y una llamada de atención contra los habituales reduccionismos:

1. Presentar situaciones problemáticas abiertas de un nivel de dificultad adecuado (correspondiente a la zona de desarrollo potencial de los y las estudiantes) con objeto de que puedan tomar decisiones para precisarlas y entrenarse así en la transformación de situaciones problemáticas abiertas en problemas precisos (ver punto 3 ).

2. Favorecer la reflexión de los estudiantes sobre la relevancia y el posible interés de las situaciones propuestas, que dé sentido a su estudio (considerando las posibles implicaciones CTS, etc.) y evite un estudio descontextualizado, socialmente neutro.

3. Potenciar los análisis cualitativos, significativos, que ayuden a comprender y acotar las situaciones planteadas (a la luz de los conocimientos disponibles, del interés del problema, etc.) y a formular preguntas operativas sobre lo que se busca.

Se trata de salir al paso de operativismos ciegos sin negar, muy al contrario, el papel esencial de las matemáticas como instrumento de investigación, que interviene en todo el proceso, desde el enunciado mismo de problemas precisos (con la necesaria formulación de preguntas operativas, etc.) hasta el análisis de los resultados.

4. Plantear la emisión de hipótesis como actividad central de la investigación científica, susceptible de orientar el tratamiento de las situaciones y de hacer explícitas, funcionalmente, las preconcepciones de los estudiantes.

Insistir en la necesidad de fundamentar dichas hipótesis y prestar atención, en ese sentido, a la actualización de los conocimientos que constituyan prerrequisitos para el estudio emprendido.

Reclamar una cuidadosa operativización de las hipótesis, es decir, la derivación de consecuencias contrastables, prestando la debida atención al control de variables, etc.

5. Conceder toda su importancia a la elaboración de diseños y a la planificación de la actividad experimental por los propios estudiantes.

Potenciar, allí donde sea posible, la incorporación de la tecnología actual a los diseños experimentales (ordenadores, electrónica, automatización...) con objeto de favorecer una visión más correcta de la actividad científico-técnica contemporánea.

6. Plantear el análisis detenido de los resultados (su interpretación física, fiabilidad, etc.), a la luz del cuerpo de conocimientos disponible, de las hipótesis manejadas y de los resultados de «otros investigadores» (otros equipos de estudiantes).

Favorecer, a la luz de los resultados, las necesarias revisiones de los diseños, de las hipótesis o, incluso, del 
planteamiento del problema. Prestar una particular atención, en su caso, a los conflictos entre los resultados y las concepciones iniciales, facilitando así, de una forma funcional, los cambios conceptuales.

7. Plantear la consideración de posibles perspectivas (replanteamiento del estudio a otro nivel de complejidad, problemas derivados...) y contemplar, en particular, las implicaciones CTS del estudio realizado (posibles aplicaciones, repercusiones negativas...).

8. Pedir un esfuerzo de integración que considere la contribución del estudio realizado a la construcción de un cuerpo coherente de conocimientos, así como las posibles implicaciones en otros campos de conocimientos.

9. Conceder una especial importancia a la elaboración de memorias científicas que reflejen el trabajo realizado y puedan servir de base para resaltar el papel de la comunicación y el debate en la actividad científica.

10. Potenciar la dimensión colectiva del trabajo científico organizando equipos de trabajo y facilitando la interacción entre cada equipo y la comunidad científica, representada en la clase por el resto de los equipos, el cuerpo de conocimientos ya construido (recogido en los textos), el profesor como experto...

Hacer ver, en particular, que los resultados de una sola persona o de un solo equipo no pueden bastar para verificar o falsar una hipótesis y que el cuerpo de conocimientos constituye la cristalización del trabajo realizado por la comunidad científica y la expresión del consenso alcanzado en un determinado momento.

Insistimos en que los aspectos contemplados no constituyen ningún algoritmo, ningún intento de ahormar la actividad científica en unos «pasos» 0 «etapas», sino un recordatorio de la riqueza del trabajo científico. Una riqueza que debe estar presente en los intentos de transformar toda la enseñanza de las ciencias y no sólo las prácticas. De hecho, la orientación propuesta cuestiona la idea de "práctica de laboratorio» como actividad autónoma, puesto que la investigación científica abarca mucho más que el trabajo experimental y éste no tiene sentido tomado aisladamente.

Terminamos aquí estas consideraciones generales y pasamos seguidamente a transcribir un ejemplo ilustrativo de la orientación propuesta.

\section{UN EJEMPLO ILUSTRATIVO: ESTUDIO DE LA CAIDA DE GRAVES}

¿Por qué hemos elegido esta práctica tan conocida y al mismo tiempo, según una opinión bastante generalizada, tan poco atractiva? ¿Qué interés pueden tener los estudiantes, hoy en día -se suele preguntar-en dejar caer bolitas por un plano inclinado? ¿En qué medida van a poder adquirir con ello una visión estimulante y actual de la ciencia? ¿Qué interés puede tener, en definitiva, esa «física prehistórica»?

Son esas mismas preguntas las que nos han movido a elegir una práctica tan «tradicionai", pues pretendemos mostrar que la falta de atractivo de este tipo de trabajos deriva de la orientación que habitualmente se les da, y que su replanteamiento como una investigación, en la forma que aquí presentaremos, puede generar auténtico interés y proporcionar también -a través de la incorporación de elementos de la tecnología moderna a los diser̃os experimentales y al tratamiento de los resultados- una visión más actual de la ciencia.

Se trata de una práctica, además, en cuya transformación habíamos trabajado ya ambos autores de forma independiente, lo que facilitaba la puesta en común de nuestras respectivas orientaciones, como una contribución a un modelo más consensuado.

A continuación reproducimos el programa de actividades que hemos concebido para orientar la investigación de los estudiantes (designadas con la notación A.1., A.2., etc.), acompañadas de comentarios que intentan justificar dichas actividades, transcribir sintéticamente las contribuciones de los estudiantes, etc. La versión aquí propuesta ha sido ensayada en el curso 1994-95 con profesores en formación de la Escuela de Magisterio Ausiàs March de la Universitat de València.

Digamos por último, antes de pasar a transcribir esta práctica de caída de graves, que su realización se propone cuando se ha procedido ya a la construcción -planteada también como una investigación dirigida (Calatayud et al. 1990)-de las magnitudes que permiten determinar la posición đe un objeto y los cambios que ésta experimenta, introduciendo los conceptos de posición, desplazamiento, velocidad y aceleración, así como las ecuaciones del movimiento que resultan en el caso de que la velocidad sea constante o lo sea la aceleración. Precisamente dichos conceptos y ecuaciones tienen un carácter de construcciones tentativas, de hipótesis de trabajo, y se trata ahora de constatar su validez para el estudio de los movimientos reales, como el de caída de los graves que aquí se propone, es decir, de constatar su capacidad para describirlos y predecir resultados contrastables experimentalmente.

\section{Consideración del posible interés de la situación planteada}

\section{A. 1. Discutir el posible interés que tiene el estudio de la caida de los cuerpos.}

Conviene insistir en la importancia de esta discusión previa acerca del interés del estudio planteado: una orientación investigativa como la que aquí se propone es incompatible con la inmersión de los estudiantes en una tarea cuya finalidad y sentido se les escape. Esto es lo que suele hacerse, sin embargo, incluso cuando existe la voluntad de plantear la tarea como una investigación. Se argumenta al respecto que los alumnos difícilmente 
podrán conocer las razones que muestran la relevancia del estudio planteado y su posible interés. De hecho, cuando se les plantea dicha reflexión en esta práctica, inicialmente apenas se les ocurre nada, más allá de algunos tópicos como «se trata de un movimiento habitual en la vida cotidiana" y otros del mismo estilo. Pero, una vez roto el «hielo inicial», va surgiendo toda una variedad de argumentos -relativos al movimiento de los proyectiles, etc.- que, además de favorecer una actitud más positiva, permiten una aproximación funcional a las relaciones CTS y favorecen la adquisición de una concepción preliminar de la tarea. Conviene puntualizar, sin embargo, que lo esencial no es que los estudiantes sean capaces de đar abundantes y valiosos argumentos sobre el interés de la situación planteada, sino que se modifique la actitud con que enfocan esta tarea, haciéndola más relevante, menos «ejercicio escolar».

El profesor tiene, claro está, un papel esencial en esta discusión: le corresponde resaltar y «amplificar» los argumentos dados por los estudiantes y añadir orros, intentando que aparezcan como derivación de los que ellos han utilizado. Asi, la idea de que «se trata de un movimiento habitual en la vida cotidiana" puede dar lugar a que el profesor resalte algunos aspectos como, en primer lugar, la importancia de recurrir a un movimiento muy común, relativamente simple y fácil de reproducir, para comenzar a estudiar la validez de los conceptos introducidos hasta aquí. Se puede insisitir, a ese respecto, en que los investigadores comienzan, en general, con el planteamiento de situaciones sencillas, acotadas, para pasar después a otras más complejas. Si queremos conocer un movimiento con importantes aplicaciones prácticas como el lanzamiento de un proyectil (por citar un ejemplo habitualmente mencionado por los estudiantes), es conveniente comenzar por la situación más elemental, que es, precisamente, la de su caída desde una cierta altura.

Por otra parte, el hecho de que se trate de un movimiento reiteradamente observado, permite también hacer una prediccion «inquietante»: su estudio permitirá constatar -puede anunciarse a los estudiantes-que muchas cosas que nos son familiares resultan sistemáticamente mal interpretadas. Ello les aproximará a una característica esencial de la actividad científica: la necesidad de cuestionar lo que parece obvio, evidente, «de sentido común". Hemos podido comprobar que una predicción como ésta, realizada con cierto énfasis, genera un cierto «suspense» y refuerza el interés del trabajo que se va a realizar.

Cabe señalar, por último, que al evaluar la nueva orientación de los trabajos prácticos, los estudiantes valoran muy positivamente esta reflexión inicial y la consideran uno de sus elementos más importantes y motivadores.

\section{Análisis cualitativo inicial de la situación y precisión del problema}

La discusion acerca de la importancia del estudio planteado contribuye, como ya hemos señalado, a que los estudiantes comiencen a formarse una concepcion preliminar de la situación problemática. Ello ahora les permite -sin la brusquedad que supone «entrar en materia» directamente- realizar un análisis cualitativo más detenido, que les ayude a acotar la situación y transformarla en un problema preciso. A tal objeto se puede plantear la actividad que describimos a continuación:

\section{A.2. Teniendo en cuenta las experiencias cotidianas, ¿qué puede decirse, a título de primeras conjeturas, acerca del movimiento de caída de los cuerpos?}

En la discusión con los alumnos aparecen dos núcleos de ideas (Gil et al., 1991):

1) Muchos de ellos piensan que mientras mayor sea la masa del cuerpo más rápidamente llegará al suelo, aunque algunos otros puedan cuestionarlo, porque recuerdan haber estudiado en algún curso precedente que el tiempo de caída es independiente de la masa.

2) Se trata de un movimiento de velocidad creciente, tal vez uniformemente acelerado.

Conviene centrarse, en primer lugar, en la hipótesis de la influencia de la masa. Como vemos, el debate ha permitido sacar a la luz, de un modo natural, Ias preconcepciones que tienen los alumnos sobre el fenómeno estudiado. Diversas investigaciones han mostrado, en efecto, to persistente que resulta la creencia, en estudiantes de distintos niveles de enseñanza, de que la rapidez de la caída depende de la masa del cuerpo. Pero estas preconcepciones adquieren ahora el estatus de hipótesis que deben ser contrastadas y, en caso de verse falsadas, sustituidas por otras, etc. Se evita así que el conflicto cognitivo entre distintas concepciones adquiera la connotación negativa que, en las estrategias de "cambio conceptual», representa la confrontacion entre las «ideas propias" de los estudiantes y las científicas (Driver, 1986).

Cuando se pide a los estudiantes que fundamenten su hipótesis, avanzan argumentos que pueden ser parcialmente ciertos (como «el cuerpo que pesa más es atraído con una fuerza mayor»), pero que conducen a conclusiones incorrectas por incurrir en «reduccionismo funcional” (es decir, por no tener en cuenta otras posibles consecuencias de la modificación de la masa). El argumento principal, sin embargo, es la experiencia reiterada de ver caer lentamente objetos muy ligeros, y con rapidez los más pesados. Es esta «evidencia» la que se impone... y la que merece ser cuestionada, sin detenerse, por ahora, en mayores fundamentaciones, que obligarían a consideraciones dinámicas prematuras y podrían debilitar el muy conveniente «choque» producido por la falsación de la hipótesis.

A.3. Proceder a contrastar las hipótesis acerca de la influencia o no de la masa en el tiempo de caída.

La experiencia que consiste en dejar caer dos cuerpos «pesados» que tienen masas muy diferentes permite a los estudiantes constatar que, en general, el tiempo de caída 
no depende de la masa, al menos de modo esencial. Pero se plantea también la điscusión de por qué razón cuerpos «muy ligeros», como una hoja de papel, una pluma, etc., caen tan lentamente, haciendo surgir la idea de que ello sea debido a la fricción con el aire. Conviene, pues, proponer la siguiente actividad.

\section{A.4. Diseñar distintas experiencias para mostrar que, si se hace despreciable la fricción con el aire, todos los cuerpos caen prácticamente en el mismo tiempo.}

Los alumnos sugieren, a menudo, la utilización de un tubo largo de vidrio del cual se pueda extraer el aire. Galileo no pudo realizar esta experiencia porque en aquella época aún no se había construido la bomba de vacío, y tampoco hoy muchas escuelas cuentan con tales bombas y con el tubo de vidrio adecuado para realizarla. Ello obliga a solicitar otros diseños, aunque valorando como se merece esta propuesta de los estudiantes, que constituye la forma de contrastación más directa.

Los estudiantes proponen entonces diversos e ingeniosos diseños para reducir la fricción de, por ejemplo, una hoja de papel con el aire, similares a los que recoge la historia de la ciencia: colocar la hoja de papel sobre un libro y dejarlos caer; hacer caer verticalmente la hoja de papel (colocándola, para ello, junto a un libro también vertical); «arrugar» la hoja de papel hasta transformarla en una pequeña esfera. Los tres diseños, y particularmente el último, llevan a la conclusión de que, en ausencia de fricción con el aire, el tiempo de caída es independiente de la masa de los cuerpos.

Los estudiantes se han visto obligados, pues, a modificar su hipótesis inicial y, al propio tiempo, a replantear la investigación, acotándola con mayor precisión: ahora se trata de estudiar la caída de los cuerpos en ausencia de fricción con el aire (o cuando ésta es despreciable). Esto es algo que merece ser resaltado, pues es una buena ocasión para que perciban el carácter no lineal de una investigación.

Nos ocuparemos, en lo que sigue, de la segunda de las hipotesis inicialmente formuladas, teniendo ahora en cuenta las precisiones introducidas sobre ausencia de friccción, lo que permite ignorar, además de la masa, la forma y extensión de los cuerpos.

\section{Operativización de la hipótesis acerca de que el movimiento de caída de los cuerpos es uniformemente acelerado}

Dado que no podemos medir directamente la aceleración de caída para comprobar si es constante o no, es necesario derivar consecuencias contrastables, que hagan dicha hipótesis operativa:

\section{A.5. Derivar, a partir de a = cte., alguna consecuencia directamente contrastable.}

Esta derivación implica el manejo del cuerpo de conocimientos disponible, poniendo de manifiesto, una vez más, el importante papel que éste juega a lo largo de toda la investigación. Los estudiantes, tras concluir que las ánicas medidas directas posibles, en el estudio de un movimiento, son las de espacios y tiempos, y habiendo deducido ya las ecuaciones del movimiento uniformemente acelerado, recurren a la ecuación e $=1 / 2 \mathrm{at}^{2}$. La hipótesis operativa es, pues, que la relación entre los tiempos $t$ de caída desde distintas alturas y los valores $h$ de dichas alturas ha de ajustarse a la ecuación $h=k t^{2}$.

Otra variante para operativizar la hipótesis consiste en construir el gráfico $v=f(t)$ con el fin de comprobar si es una linea recta. Ello remite también, por supuesto, a medidas de espacios y tiempos y, por otra parte, resulta menos claro para los estudiantes. Puede ser interesante, sin embargo, mostrar que existen distintos caminos de contrastación, distintas consecuencias contrastables.

\section{Elaboración de estrategias para comprobar la hipó- tesis formulada}

A.6. Diseñar experimentos para contrastar la hipótesis de que el movimiento de caída de los cuerpos es uniformemente acelerado.

Los estudiantes, de entrada, suelen proponer dejar caer una pequeña esfera desde distintas alturas y medir en cada caso el tímpo empleado en caer para ver si los valores obtenidos se ajustan a la relación prevista. Es oportuno hacer notar que los tiempos de caída son tan pequeños que no es posible realizar medidas precisas de los mismos en esas condiciones. Los estudiantes conciben entonces la posibilidad de fotografiar la caída de la esfera, al lado de una cinta métrica, "con una cámara que dispare automáticamente a intervalos de tiempo regulares y muy breves». Éste es un procedimiento que se ha utilizado, con buenos resultados - conviene indicarlo a los estudiantes a modo de refuerzo de sus planteamientos-recurriendo a fotografías estroboscópicas.

La idea de la automatización aparece como algo básico para evitar los problemas de coordinación entre el instante de soltar la esferita y la puesta en marcha del cronómetro. En ese sentido surge también la propuesta de utilizar relojes electrónicos, que se pongan en marcha al soltarse la esfera y se paren al chocar ésta contra un tope.

Aquí es pertinente señalar que en calidad de reloj electrónico puede emplearse un ordenador, to que permitiría, además, elevar el nivel de automatización en la realización del experimento (Gallego, Lowy y Robles, 1991). En particular, teniendo en cuenta su capacidad para almacenar datos en memoria, parece lógico intentar, utilizando determinados sensores, el registro de las distancias y los tiempos en un movimiento único, evitando así la necesidad de repetir varias veces las experiencias de caída. Por otra parte, el disponer de los datos en la memoria del ordenador posibilitaría, mediante un programa informático, profesional o elaborado al efecto, el procesamiento inmediato de ellos. Estas ideas merecen ser resaltadas como ejemplos de aproximación a los 
principios tecnológicos de la automatización de experimentos, lo cual debe constituir uno de los objetivos de la enseñanza de las ciencias en la actualidad (VaIdés y Valdés, 1994).

Pese al interés de las propuestas precedentes, conviene hacer notar a los estudiantes que en la época de Galileo no se disponía, obviamente, de medios adecuados ni para la medida precisa de los tiempos ni para la automatización. Ello le llevó a concebir la posibilidad de «debilitar» la caída, haciéndola más lenta. Se trataba de imaginar algún movimiento asociado a la caida de los cuerpos pero que tuviera lugar más lentamente (sin para ello, claro esta, introducir fricción). Esto constituye una estrategia ingeniosa para salir del «impase» y merece la pena que los estudiantes se planteen dicha tarea como un ejemplo de la creatividad que exige, en todo momento, el desarrollo de una investigación:

\section{A.7. Concebir varios procedimientos para "debilitar» la caida de los cuerpos pero sin desvirtuar su naturaleza de caída en ausencia de fricción.}

Cabe señalar que, en ocasiones, algunos estudiantes conocen ya el experimento del plano inclinado, de aquí que la actividad solicite varios procedimientos. Los estudiantes encuentran serias dificultades para imaginar un diseño adecuado y sus primeras propuestas suelen introducir fricción («dejar caer la esferita en un tubo Ileno de un líquido viscoso"; "colgar la esferita de un pequeño paracaídas"...). La discusión de estas propuestas y la insistencia del profesor en que se trata de lograr que el cuerpo caiga más lentamente sin introducir fricción conduce, sin embargo, a propuestas adecuadas -además de dejar caer la esferita por un plano inclinadocomo, por ejemplo, colgar dos masas iguales de los extremos de un cordel que pasa por una polea sin rozamiento apreciable y colocar una pequeña sobrecarga en uno de los extremos. Otro diseño parecido y habitualmente propuesto consiste en utilizar un carrito que pueda moverse por un plano horizontal con fricción despreciable, del que tira -con ayuda de una cuerda y polea fija al extremo del plano- una pequeña masa que cae verticalmente.

Es preciso insistir en que merece la pena tener algo de paciencia y permitir a los estudiantes que lleguen a concebir estos diferentes diseños, pues ello constituye una excelente ocasión para que entren en contacto con una de las tareas más creativas y satisfactorias del trabajo científico (lamentablemente escamoteada en las prácticas habituales, cuyo diseño se da ya elaborado).

Se puede proceder ahora a realizar algunos de los experimentos diseñados sin el peligro de que sean vistos como tareas tediosas, sin interés y sin vinculación con lo que es la ciencia actual.

\section{Planificación y realización de los experimentos}

Aunque al llegar a este punto se posee ya una concepción general de los diseños, ello no significa que ahora quede una actividad puramente manipulativa:
A.8. Proceder a la realización del experimento relativo a la caida de una esferita por un plano inclinado.

Incluso en un diseño tan elemental como éste, desđe el punto de vista técnico, surgen numerosos problemas que deben ser resueltos. Por ejemplo, ¿cómo soltar la esfera para no comunicarle velocidad inicial? o ¿cómo coordinar el inicio y final del movimiento con la puesta en marcha y detención del cronómetro? La dificultad de esta coordinación-que se traduce en dispersiones muy significativas de los tiempos, dada su brevedad- hace ver la conveniencia de automatizar el proceso, por ejemplo, con ayuda de un ordenador. Esto requiere -si los estudiantes carecen de experiencia en este campo- una intervención mucho más directa del profesor, pero la comprensión de los montajes y programas informáticos necesarios está al alcance de los estudiantes y permite la vinculación de esta investigación con elementos fundamentales de la tecnología moderna.

Utilizando la funcion TIMER del lenguaje BASIC, por ejemplo, es posible medir intervalos de tiempo con exactitud de unas cinco centésimas de segundo, to que seria suficiente en el caso de la caída por un plano inclinado; y mediante un programa convenientemente elaborado dicha exactitud puede llegar hasta $10^{-5} \mathrm{~s}$. La entrada de informacion digital al ordenador se efectúa empleando sencillos interruptores: por ejemplo, la esfera puede estar cerrando inicialmente un circuito conectado al ordenador y al soltarla, es decir, al abrir el circuito, se pone en marcha el reloj; luego, cuando la esferita choca contra un tope móvil, provocando la apertura de otro circuito, se realiza la lectura del tiempo transcurrido. El registro de la información puede hacerse a través del puerto de juegos mediante la función INP.

No es necesario, sin embargo, proceder en este momento a un estudio detenido de todo el proceso de automatización, programas informáticos, etc. Ello constituye, en sí mismo, una investigación tan exigente o más que el estudio del movimiento a que estamos procediendo. Por eso pueđe ser más adecuado aquí limitarse a utilizar los medios disponibles y dejar planteado, como perspectiva futura, el estudio detenido de sus fundamentos, aplicaciones generales, etc. Se trataría, pues, de presentar brevemente a los estudiantes el montaje que va a utilizarse -siguiendo su propuesta de automatización-y pedirles la realización del experimento, que ahora puede ser, directamente, la caída vertical, gracias a la mayor precisión alcanzada en la medida de los tiempos.

A.9. Proceder a la realización del experimento relativo a la caida vertical, automatizando las mediciones de tiempo con ayuda de un ordenador.

Por último, si se dispone del equipo que permita obtener la fotografía estroboscópica, se puede proceder a la realización de este experimento, aunque plantea dificultades que no son fáciles de resolver en el aula. Es posible, sin embargo, proporcionar a los estudiantes la fotografía obtenida "por ofros investigadores». 
A.10. La figura adjunta muestra la fotografía estroboscópica de una esferita que se dejó caer desde cierta altura. Proceded a la construcción de una tabla de espacio vs. tiempo.

Los estudiantes han de efectuar la lectura cuidadosa de las distancias recorridas por la esferita (con ayuda de la cinta métrica que aparece en la misma foto) y el cálculo de los tiempos correspondientes.

Ésta puede ser una buena ocasión para recordar que la verificación de una hipótesis implica, en general, el trabajo de numerosos equipos, y que no tiene sentido pensar que un sólo equipo ha de realizar todos los experimentos posibles. Lo que sí es necesario es poner en común los distintos resultados obtenidos y constatar en qué medida son coherentes entre sí. Ello nos remite, pues, al análisis de los resultados.

\section{Análisis y comunicación de los resultados y de las perspectivas abiertas}

\section{A.11. Analizar e interpretar los resultados obtenidos en los experimentos realizados.}

Los resultados obtenidos con el plano inclinado utilizando un cronómetro manual parecen ajustarse a la relación $\mathrm{e}=\mathrm{kt}^{2}$, aunque con elevados márgenes de imprecisión. La automatización de la medición del tiempo mejora muy sensiblemente esos resultados, incluso para la caida vertical desde pequeñas alturas. En este caso el gráfico de $e=f\left(t^{2}\right)$ es una clara línea recta, sin apenas desviaciones. Lo mismo ocurre con los valores que se obtienen a partir de la fotografía estroboscópica.

Se puede ir un poco más lejos en el análisis de los resultados y solicitar a los estudiantes que determinen el valor de la aceleración de cáda libre y que lo cotejen con el que se proporciona en los libros de texto.

\section{A.12. Determinar el valor de la aceleración de caída libre de un cuerpo a partir de los datos obtenidos (actividades A.9 O A.1O).}

Los valores obtenidos para esta aceleración son, en general, muy próximos al valor aceptado por la comunidad científica, lo cual tiene un efecto particularmente motivante para los estudiantes.

Todos los resultados apoyan, pues, la hipotesis de la aceleración de caída constante. Esta era, por lo demás, la hipótesis inicial. Podría pensarse, por ello, que quizás no era necesario un tratamiento tan detenido y que una simple verificación con un único experimento bastaba. Sin embargo, es preciso dejar bien patente que la aceptación de un resultado por la comunidad científica tiene muy serias exigencias que obligan a la obtención de una multiplicidad de resultados en distintas situaciones y a mostrar la coherencia de todos ellos. Ésta es la mejor forma de romper con aceptaciones acríticas de las «evidencias de sentido común» como, por ejemplo, la creencia de que los cuerpos caen tanto más aprisa cuanto mayor es su masa. Debemos ser conscientes, a este respecto, de que, aunque dicha hipótesis ha sido claramente falsada con los experimentos realizados en la primera parte de esta investigación, la superación permanente de estas ideas espontáneas no puede ser el resultado de algunos experimentos como los realizados, sino que exige la adquisición de un cuerpo de conocimientos coherente y global y, más aún, una nueva forma de razonar, de enfrentarse a los problemas.

De hecho, los estudiantes no pueden explicarse por qué cuerpos de distinta masa caen con la misma aceleración; y no lo harán mientras no se apropien del sistema de conceptos de la mecánica newtoniana. Por ello, a pesar de los resultados obtenidos en esta investigación, bastantes estudiantes vuelven a utilizar sus esquemas iniciales en cuanto se varía ligeramente el contexto. Dicho de otro modo, los cambios conceptuales no se producen con tratamientos puntuales, sino como resuitado de la adquisición de un cuerpo de conocimientos capaz de desplazar, de forma global, las concepciones iniciales. Esto es algo que debe quedar claro al discutir las perspectivas abiertas por la investigación.

\section{A.13. Considerar las perspectivas abiertas por esta investigación susceptibles de originar nuevos estudios.}

Muchas de las perspectivas han sido considezadas ya en Ios momentos oportunos durante el desarrollo de la investigación, pero al finalizar ésta conviene recapitularlas. Podemos referimos así, entre otras tareas que han quedado pendientes, a:

- explicar el hecho de que en ausencia de fricción con el aire todos los cuerpos caen con la misma aceleración;

- extender la investigación al estudio de otros movimientos de interés práctico, como el de los proyectiles, etc.;

- investigar los factores de los cuales depende la resistencia que ofrece el aire durante la caída de un cuerpo;

- diseñar dispositivos que permitan elevar el nivel de automatización del registro de posiciones y tiempos durante el movimiento de un cuerpo, profundizando en las características de distintos tipos de sensores y en el funcionamiento, a este respecto, de un ordenador, diseñando sencillos programas que posibiliten el registro de información digital, etc.

Conviene, por último, que los estudiantes recojan el trabajo realizado en una memoria sobre la investigacion, planteada como práctica de un aspecto esencial de la actividad científica: la comunicación.

A.14. Elaborar una memoria de la investigación realizada, destinada a ser publicada en las actas del curso.

Es preciso dar a esta actividad el sentido de la auténtica comunicación científica, superando su connotación habitual de simple ejercicio escolar, destinado a ser calificado por el profesor. En este sentido, puede ser muy 
conveniente hacer jugar el papel de «referees" a cada grupo de estudiantes, dándoles a analizar un cierto número de memorias para que sugieran modificaciones a los autores, etc. La «publicación» de unas actas del trabajo resalizado durante el curso y la organización de sesiones de comunicación oral (con ayuda de transparencias, etc.) y de sesiones póster contribuyen a dar interés a este esfuerzo de comunicación además de proporcionar una visión más correcta del trabajo científíco, buena parte del cual está centrado en dicha comunicación.

\section{NOTA FINAL}

La orientación de los trabajos prácticos que hemos ilustrado con este ejemplo pretende que los estudiantes se familiaricen con la extraordinaria riqueza de la actividad científica, superando los reduccionismos habituales. Es conveniente, por ello, terminar solicitando una recapitulación de los aspectos más destacados del tratamiento realizado, con objeto de favorecer una metareflexión que refuerce la apropiación consciente đe las estrategias del trabajo científico.

Puede ser conveniente también que los profesores procedamos a đicha recapitulación en cada práctica que prepa-

\section{REFERENCIAS BIBLIOGRÁFICAS}

BELL, B. y PEARSON, J. (1992). Better Learning. International Journal of Science Education, 14(3), pp. 349-361.

CAAMAÑO, A., CARRASCOSA, J, y OÑORBE, A. (1994). Los trabrajos prácticos en las Ciencias Experimentales, Alambique. Didactica de las ciencias experimentales, $2, \mathrm{pp}$. 4-5.

CALATAYUD, M. ctal. (1990). Laconstrucción de las ciencias físico-químicas. Valencia: NAU llibres.

DÉSAUTELS, J., LAROCHELLE, M., CAGNÉ, B. y RUEL, F. (1993). La formation à l'enseignement des sciences: le virage épistémologique. Didaskalia, 1, pp. 49-67.

DRIVER, R. (1986). Psicología cognoscitiva y esquemas conceptuales de Ios alumnos. Enseñanza de las Ciencias, 4(1), pp. 3-15.

DUSHL, R. y GITOMER, D. (1991). Epistemological perspectives on concegtual change: implications for educational practice. Journal of Research in Science Teaching, 28(9), pp. 839-858.

GALILEGO. A., LOWY, E. y ROBLES, J. (1991). Laboratorio asistido por ordenador. Madrid: Phywe España. remos, tanto para poder apoyar la recapitulación que realicen los estudiantes como para analizar si el programa de actividades, diseñado para dirigir la investigación, es adecuado para proporcionar una visión correcta de la ciencia como actividad abierta y creativa.

Invitamos a los lectores a realizar dicha recapitulación para el ejemplo que hemos transcrito, con la esperanza de que el resultado les parezca una propuesta inicialmente aceptable y suficientemente abierta para permitir su apropiación personal y perfeccionamiento, en un proceso que convierte también el trabajo docente en una actividad investigativa.

\section{AGRADECIMIENTOS}

Los autores desean expresar su agradecimiento a la Dirección General de Investigación Cientifica y Técnica (DGICYT), cuya ayuda ha hecho posible la estancia del Dr. Valdés en la Universitat de València como profesor invitado, para el desarrollo del programa de investigación «La fusión de los problemas de lápiz y papel y de las prácticas de laboratorio en actividades de investigación dirigida, siguiendo las estrategias del trabajo científico».
GIL, D. (1993). Contribución de la historia y filosofía de las ciencias ai desarrollo de un modelo de enseñanza-aprendizaje como investigación. Enseñanza de las Ciencias, 11(2), pp. 197-2 12.

GIL, D. (1994), Relaciones entre conocimiento escolar y conocimiento científico. Investigación en la Escuela, 23, pp. 17-32.

GIL, D. y CARRASCOSA, J. (1985). Science leaming as a conceptual and methodologicat change. European Journal of Science Education, 7(3), pp. 231-236.

GIL, D., CARRASCOSA, J., FURIÓ, C. y MARTÍNEZ. TORREGROSA, I. (1991). La enseñanza de las ciencias en la educación secundaria. Barcelona: Horsori

GIL, D., NAVARRO, J. y GONZÁLEZ, E. ( 1993 ). Las prácticas de laboratorio en la formación del profesorado (II). Una expertencia de transformación de las prácticas del ciclo básico universitario. Revista de Enseñanza de la Física, 7(1), pp. 33-47.

GONZÁLEZ, E. (1992). ¿Qué hay que renovar en los trabajos prácticos?, Enseñanza de las Ciencias, 10(2), pp. 206-211. 
GONZÁLEZ, E. (1994). Las prácticas de laboratorio en la formación del profesorado de física. Tesis doctoral: Departament de Didactica de les Ciències Experimentals, Universitat de València.

GRAU, R. (1994). ¿Qué es lo que hace difícil una investigación? Alambique. Didáctica de las Ciencias Experimentales, 2, pp. 27-35.

HASHWEH, M. (1986). Towards an explanation of conceptual change. European Journal of Science Education, 8(3), pp. 229-249.

HODSON, D. (1992). In search of a meaningful relationship: an exploration of some issues relating to integration in science and science education. International Journal of Science Education, 14(5), pp. 541-566.

HODSON, D. (1992b). Assessment of practical work: some considerations in Philosophy of Science. Science \& Education, 1 , pp. 115-144.

HODSON, D. (1993). Re-thinking old ways: towards more critical approaches to practical work in school science. Studies in Science Education, 22, pp. 85-142.

HODSON, D. (1994). Hacia un enfoque más crítico del trabajo de laboratorio. Enseñanza de las Ciencias, 12(3), pp. 299 313.

LILLO, J. (1994). Los trabajos prácticos de ciencias naturales como actividad reflexiva, crífica y creativa, Alambique. Didáctica de las Ciencias Experimentales, 2, pp. 47-56.

MEICHSTRY, Y. (1993). The impact of science curricula on students views about the nature of science. Journal of Research in Science Teaching, 39(5), pp. 429-443.
NIEDA, J. (1994). Algunas minucias sobre los trabajos prácticos en la enseñanza secundaria, Alambiaue. Didáctica de las Ciencias Experimentales, 2, pp. 15-20.

PAYÁ, J. (1991). Los trabajos prácticos en la enseñanza de la física y quimica: un análisis crítico y una propuesta fundamentada. Tesis doctoral: Departament de Didactica de les Ciències Experimentals, Universitat de València.

PRAIA, I. y CACHAPUZ, F. (1994). Un análisis de las concepciones acerca de la naturaleza del conocimiento científico de los profesores portugueses de la enseñanza secundaria. Enseñanza de las Ciencias, 12(3), pp. 350-354.

SALINAS, J. (1994). Las prácticas de física básica en laboratorios universitarios. Tesis doctoral: Departament de Didàctica de les Ciències Experimentals, Universitat de Vałència.

TAMIR, P. y GARCIA, M. (1992). Características de los ejercicios de prácticas de laboratorio incluidos en los libros de textos de ciencias utilizados en Cataluña. Enseñanza de las Ciencias, 10(1), pp. 3-12.

VALDÉS, R. y VALDÉS, P. ( 1994). Utilización de los ordenadores en la enseñanza de las ciencias, Enseñanza de las Ciencias, $12(3)$, pp. 412.415

WATSON, J. (1994). Diseño y realización de investigaciones en las clases de ciencias, Alambique. Didáctica de las Ciencias Experimentales, 2, pp. 57-65.

WHEATLEY, G. (1991). Constructivist perspectives on Science and Mathematics learning, Science Education, 75(1), pp. 9-2l.

[Artículo recibido en junio de 1995 y aceptado en enero de 1996. ] 


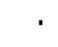

